\title{
Index to Volume 79
}

Accidental drowning, 331-33

Adams, John, 217

Adams, John Quincy, 217

Adolescence, 311-41; beginning of, 316, 340-41; biological definition, 314-15; in the eastern U.S., 314-15, $317,329,337,339,341$; end of, 316$17,340-41$

Adolescent employment, 324-28; table illustrating, 325, 338

Adolescent self-determination, 329331

Advice literature (books and pamphlets), 312-13, 315

African Americans, 36, 41, 47, 141, 157, 237-38, 259, 360-61

Age-graded public schools, 313, 316, 341

Age of consent, 53

Age of Fear: Othering and American Identity during World War I, reviewed, 100-02

Age of majority, 45, 53, 313-16, 320, 322-23, 331, 340

The Alchemy of Slavery: Human Bondage and Emancipation in the Illinois Country, 1730-1865, reviewed, 8587

Alexander, Thomas G., book by, reviewed, 292-94

Aley, Ginette, book review by, 30001

Alger, Horatio, 136

Allosso, Dan, book by, reviewed, 386-88

Amana Colonies, 176

America Ascendant: The Rise of American Exceptionalism, reviewed, 398400

American Dream, 344, 366

American exceptionalism, 174-75

American history (subject), 220, 226$31,233,240-42$
American Legion, 245

American Library Association, 131, $139,146,148,151$

American Republic Insurance, 161, 171

American Revolution, 354-55

Americanism, 217, 226-27, 231-32, 244-45

Ames, Iowa, 343-66; population of, 349-52

Ames Chamber of Commerce, 35556

Ames City Council, 351-53, 355, 359-62

Ames City Directory, 360

Ames Daily Intelligencer, 347

Ames Daily Tribune, 343, 345, 352-55, 360-63

Ames High School, 352

Ames Industrial Development, Inc., 355-56

Ames League of Women Voters, 35556

Anderson, Douglas Firth, book review by, 199-201

Anderson, Gary Clayton, book by, reviewed, 380-82

Anonymity, 248, 254, 259-60, 268, 273

Anthony, Susan B., 43

Anticatholicism, 259, 262-68, 274, 280

Anticommunist (American) propaganda, 157, 173, 182, 185

Anti-public school activism, 261, 314

Antisuffragists, 42-43

Applied patriotism, 231-34, 242-43

Apprenticeship, 316-17, 324-25, 327$28,330,334,337-38$

Apps, Jerry, book by, reviewed, 10608

Armstrong, Louis, 178

Aron, Raymond, 156 
Arson, 261-62, 268-69

The Athlete's Guide, 58

Austen, Jane, 141

Authorized Agents: Publication and Diplomacy in the Era of Indian Removal, reviewed, 190-92

Avant-Garde in the Cornfields: Architecture, Landscape, and Preservation in New Harmony, reviewed, 308-10

Baby Boom, 343

Barbells, 56, 65-66, 71, 78, 83

Barber, Marilyn, 329-30

Baseball, 70-7, 74, 78

Basketball players, 68-70, 81; image of, 69,70

Bass, Patrick, book review by, 298300

Beardshear, William M., 212

Beauregard, Robert, 344, 354, 365

Beck, Joseph, 38

Beck, Paul N., book review by, 30305

Beienburg, Sean, book by, reviewed, 102-04

Bell, A.F., 35-42, 53

Beltman, Brian W., book by, reviewed, 199-201

Benn, David W., book review by, 189-90

Bennett, Eric, 165-66

Bernstein, Leonard, 178

Betten, Antonie, 34

Bibliotherapy, 141, 147-48, 151

Big Sioux River, 1, 21, 23

Bilasch, Theodore, 332

The Bird's Christmas Carol, 141

Blake, John Joseph, 329, 335-36

Blake, William, 335-36

Bloemendaal, E. J. G., 15

Bloody shirt, 245

Board of Control of State Institutions, 121, 123, 125-32, 135, 149-50

Bolks, Seine, 19

The Bone and Sinew of the Land: America's Forgotten Black Pioneers and the Struggle for Equality, reviewed, 192-94

Books as therapy, 141, 147-48, 151
Boone, Black Hawk, and Crockett in 1833: Unsettling the Mythic West, reviewed, 290-92

Bootlegging, 249, 260, 262-68, 281

Bowes, John P., book review by, 190 92

Bowler, Kate, book by, reviewed, 400-01

Boyd, Willard L. "Sandy," 166; book by, reviewed, 203-05

Breeders Association of Story County, 347

Bremer, Jeff, book review by, 386-88

Brigham, Johnson, 126; image of, 126

Brigham Young and the Expansion of the Mormon Faith, reviewed, 29294

Brown, Eliza H., 49-53

Brown, Thomas J., book by, reviewed, 305-06

Browning, Judkin, book co-authored by, reviewed, 384-86

Brummelkamp, Antonie, 4-5

Buchanan, Rex C., book co-authored by, reviewed, 189-90

Bucharest, Romania, 164

Buck Construction, 357-58

Budapest, Hungary, 164

Buena Vista County, Iowa, 247-83

Buena Vista County Board of Supervisors, 268-69

Buffalo Bill Cody: A Man of the West, reviewed, 95-96

Bulgaria, 164

Calliope, Iowa, 21-27, 31, 33; raid on, 23-25

Campaign for Wilson's Creek: The Fight for Missouri Begins, reviewed, 9293

Campney, Brent M.S., book by, reviewed, 376-78

Capen, Edward, 66-68, 71, 79-80

Captain M., 361

Carey, Jack, 274, 276, 279

Carey, Miriam E., 121-22, 129-32, 138-42, 144-50; image of, 130

Carney Hall, 260-62

Carr, Jo Ann Daly, book edited by, reviewed, 300-01

Carver, George Washington, 360-61 
Catalogue and Rules for Prison Libraries, 131

Catholics, 259, 262-68, 274, 280, 313, 328-29, 335-36

Catt, Carrie Chapman, 41

Cavenaugh, Catherine, 44-47

Cedar Rapids, Iowa, 356

Censorship in libraries, 135-37

Central Intelligence Agency, 165-66

Cerro Gordo County, Iowa, 240

Charis-Carlson, Jeff, book review by, 111-13

Chastity, 35-38, 41, 44-46

Chemical Lands: Pesticides, Aerial Spraying, and Health in North America's Grasslands Since 1945, reviewed, 108-09

Cherokee County, Iowa, 17-18

Cherokee Mental Health Hospital, $129,137,153,270-71$

Chicago, Illinois, 348

Chicago and North Western railroad, 356

Child labor, 312, 322

China, 165

Chui, Edward, 66-68, 79

Civic virtue, 216-17, 231

Civil Rights Era, 361

Civil War, 13-14, 16-17, 44, 230; memory of, 212-15, 220, 230, 233, 238, 245-46, 327-28

Civil War Congress and the Creation of Modern America: A Revolution on the Home Front, reviewed, 93-94

Civil War Monuments and the Militarization of America, reviewed, 305-06

The Civilian Conservation Corps in Wisconsin: Nature's Army at Work, reviewed, 106-08

Clarinda Mental Health Hospital, 129,144

Clarke, Dustin S., article co-authored by, $311-41$

Claussen, H.R., 42

Clemmons, Linda, book review by, 380-82

Cleveland, Grover, 226-27

Cliburn, Van, 178

Clinton County, Iowa, 46-47

Clow, Richmond L., 198-99

Code of Iowa, 352-53, 356-57
Colavito, Jason, book by, reviewed, 371-73

Cold War, 153-85, 344-45

Cold War liberalism, 171-73

Cole, Perry O., 240

"Colony before Party: The Ethnic Origins of Sioux County's Political Tradition," 1-34

Columbia University, 61

Compulsory school attendance laws, 312-14, 322, 341

Conard, Rebecca, book review by, 106-08, 113-14, 373-75

Conference of Natural Beauty, 359

Congressional Library, 223

Connon, David, book by, reviewed, 301-03

Conzett, Josiah, 311-12, 317-18, 323, $326,329,331-32,334-35$

Conzett, Nellie, 311-12, 317-18, 32930

Copperheads (political slang), 13

County attorney, 246-83; distinction from prosecutor, 251

Courtwright, Julie, book review by, 108-09

Cownie, John, 125, 127

Cox, Anna-Lisa, book by, reviewed, 192-94

Cox, Steven, book co-authored by, reviewed, 393-95

Cracow, Poland, 163, 181

"Criminal Seduction and Women's Citizenship in Iowa, 1865-1879," 35-55

Crumbacker, William P., 132, 136, 144

Csaplár, Vilmos, 179

Cultural diplomacy, 153-85; definition of, 155-56

Curtis, Florence Rising, 135-37

Cutter, Barbara, book review by, 382-84

Czechoslovakia, 164

Daily Iowa State Register, 43, 54

Dakota Territory, 2, 23

Dakota tribe, 18

Davis, McKinley “Deacon," image of, 69

Day, James, 50-52 
De Witt, Petra, book review by, 29698

Deere \& Company, 161-62, 176

DeLand, James, 256-58, 264

Delaware County, Iowa, 49-50

A Delicate Aggression: Savagery and Survival in the Iowa Writers' Workshop, reviewed, 111-13

Dell, Charles, 326

Delton, Jennifer, book review by, 391-93

Democracy and Education, 244

Democratic Party, 9-16, 21, 26-27, 30, 34

Demos, John, 316

Demos, Virginia, 316

Des Moines, Iowa, 11, 15, 25-26, 47, 163

Des Moines Public Library, 148

Developed land, definitions of, 344, 352-53, 358

Dewey, John, 244

Dickins, Charles, 140-41

Dickinson, North Dakota, 58-59

Doran, Walter A., 236

Dowling, David O., book by, reviewed, 111-13

Doyle, Chris, 82

Dubuque, Iowa (city), 311-41

Dubuque County, Iowa, 311-41

Dubuque Board of Education, 322

Dubuque Emigrating Company to California, 330

Duroc hogs, 347

Dutch settlements, 1-34; assimilation, 3, 6-7, 9, 12, 26-28; immigration, 3-8, 12, 16-17; opposition to, 12-13, 21-27, 32-33; politics, 9-34

East Coast, 312, 314-15, 317, 329, 337, $339,341,345$

East Germany, 156, 181

Eberle, Mark E., book by, reviewed, 95-98

Efford, Alison Clark, book review by, 98-100

Eisenhower, Dwight, 75

Eldora, Iowa, 129, 143, 145

Ellickson, Robert, 252, 271-73
Embattled Freedom: Journeys through the Civil War's Slave Refugee Camps, reviewed, 196-97

EMC Insurance, 162

The End of the Myth From the Frontier to the Border Wall in the Mind of America, reviewed, 367-69

Engle, Hualing Nieh, see Hualing Nieh

Engle, Paul, 160, 162-83; image of, 163; retirement, 178

Entireties doctrine, 256-57

An Environmental History of the Civil War, reviewed, 384-86

Equality at the Ballot Box: Votes for Women on the Northern Great Plains, reviewed, 201-03

Esplin, Scott C., book by, reviewed, 87-89

Etcheson, Nicole, book review by, 288-90

European Superiority Complex, 156, $166,174,177$

Evangelical Protestantism, 313, 316, 335

Exxon Corp, 161

Fair Housing Commission (Ames), 362

Fair Housing Ordinance (Ames), 361-62

Fair View Farm, 343-366; image of, 359

Federal Highway Act, 348

Federal Housing Administration, 365

The Federalist Frontier: Settler Politics in the Old Northwest, 1783-1840, reviewed, 288-90

Felch, Alpheus, 10

Filene, Benjamin, book review by, 104-06

Fines, 266-27

Finkelman, Paul, book co-edited by, reviewed, 93-94

Florence Crittenden Home for Unwed Mothers, 270-71

Football, 56, 60, 67, 72-78, 81-82

Ford Foundation, 158, 161, 167

Foreign Leaders Program, 158 
Fort Des Moines, 146-48; image of, 147

Fort Dodge, Iowa, 248

Fort Madison Penitentiary, 129

Franklin, Benjamin, 217

Free Democracy Party, 10

Free School Act, 14

Free Soil Party, 10-11

"The Free Voter" pamphlet, 30-33

Free will, 313, 335

Frese, Stephen J., book review by, 109-11

Friedrich and Sons Construction, 357-59

Friedrich, Bob, Jr., 363

Friedrich, Kurt, 363

Friedrich, Reinhard, Jr., 361-62, 364

Friedrich, Reinhard, Sr., 359-60, 363

"From Fair View Farm to Parkview Heights: Involuntary Annexation and the Middle-American Dream," 343-66

The Frontier Army: Episodes from Dakota and the West, reviewed, 303-05

Fulbright Program, 158, 162, 177, 182

Fulbright, William J., 177

Functional strength, 62-63, 83

Galena, Illinois, 323, 332, 334

Galush, William J., book review by, 306-08

Garfield, James A., 218

Garrison, Zachary Stuart, book by, reviewed, 296-98

Garth, Richard, 68-69

Gear, Clarissa, 323, 332, 334-35

Gear, Maria, 323, 334

Georgetown University, 247

Gergely, Ágnes, 175

German Americans on the Middle Border: From Antislavery to Reconciliation, 1830-1877, 296-98

Germans in Illinois, reviewed, 98-100

G.I. Bill, see Servicemen's Readjustment Act

Giddle, Elmer, 274-81

Gitchie Manitou the Mighty, 18

Globalism, 170-71

Glymph, Thavolia, book by, reviewed, 382-84

Godwin, P.M., 263-64
Golden Jubilee Pageant, 1-2, 5-7, 1819

Grand Army Advocate, 236

Grand Army of the Republic, 211-46

Grandin, Greg, book by, reviewed, 367-69

Granger, Barlow, 10

Grant, Ulysses S., 218

The Great Depression, 348-49, 365

Great River City: How the Mississippi Shaped St. Louis, reviewed, 369-71

Griggs, Burke W., book co-authored by, reviewed, 189-90

Grimes, James, 10

Guiler, Thomas A., book review by, 308-10

Gunkel, Ann Hetzel, book co-authored by, reviewed, 306-08

$\mathrm{H}$ and $\mathrm{F}$ Builders, 357-58

Habitat for Humanity, 361-64

Hajdik, Anna Thompson, book review by, 87-89

Hall, Greg, book review by, 393-95

Hämämläinen, Pekka, book by, reviewed, 286-88

Haraszti, Miklós, 180

Harbour, Jennifer, book review by, 192-94

Harris, M. Keith, 215

"Harvard of the West," 223

Harvard University, 60, 62, 223

Haudenshield, Jacob, 325

Hausmann, Stephen, book review by, $286-88$

Haven, Norman, 48-49

Hayes, Rutherfod B., 218

Haygood, Nancy, 35-38, 40, 53

"He Can't Vote," 354

The Heartland: An American History, reviewed, 185-87

Heemstra, Tjeerd, 22-23

Heerman, M. Scott, book by, reviewed, 85-87

Hendrickson, Paul, book by, reviewed, 390-91

Henry Ford's Five-Dollar Day, 349

Henry, O., 141

Hevel, Michael S., book review by, 203-05 
The History of Starved Rock, reviewed, 373-75

History of Story County, 346

Hoffman, Merritt, 247-48

Hoffman, Peter, 326, 333, 335

Hoganson, Kristin L., book by, reviewed, 187-89

Holland, Michigan, 9-10

Homestead Act of 1862, 14, 18, 29

Hommerding, Christopher, book review by, 96-98

Homosexuality, 273-80

Hospers, Henry, 3, 8, 10, 16-17, 20; image of, 20,21-28

Hostile Heartland: Racism, Repression, and Resistance in the Midwest, reviewed, 376-78

"'How about Some Muscle?': C.H. McCloy and Strength Training Research at the University of Iowa, 1940-1959, 56-83

Howard, Ashley, book review by, 376-78

Howell, Ronald, book by, reviewed, 395-96

Hungary, 155, 163-64

Hunter, Edith M., article by, 343-66; book review by, 305-06

In God's Presence: Chaplains, Missionaries, and Religious Space during the American Civil War, reviewed, 19495

Independence Mental Health Hospital, 129, 132, 144

Indianapolis, Indiana, 222

Industrial School for Boys, 129, 13536, 143-45

Industrial School for Girls, 129, 141, 143-45

Ingraham, Prentiss, book by, reviewed, 95

Insiders in Buena Vista County, 254$62,272-73,277-78,280$

Institution for Feeble-Minded Children, 128-29, 137, 270-71

Institute for International Education, 161,176

Institute of Cultural Relations (Hungary), 163, 165, 172
The Interior Borderlands: Regional Identity in the Midwest and Great Plains, reviewed, 285-86

Interstate Highway Act, 365

Involuntary annexation, 343-366

Iowa City, Iowa, 153-54, 161-64, 166, 170-184

Iowa City high school class, image of, 339

Iowa Confederates in the Civil War, reviewed, 301-03

Iowa Department of Transportation, 351

Iowa Federation of Women's Clubs, 124

Iowa General Assembly, 11, 14-16, 25,233

Iowa Highway Commission, 348-49, 351

Iowa International Writing Program, 153-85; funding, 164-67; uniqueness of program, 168-70

Iowa Library Association, 124

Iowa Library Commission, 124, 131, 133,150

Iowa Library Quarterly, 150

Iowa Soldiers' Orphans' Home, 12830

Iowa Star, 10

Iowa State College, 212, 347, 349-51, 360-61

Iowa State College Dairy Industries Building, 349

Iowa State Traveling Library, 150

Iowa State University, 351, 356, 36061

Iowa Supreme Court, 36-54, 356

Iowa Woman Suffrage Association, $43-44,47$

IREX, 158

Iron Curtain, 162, 178-79, 184

Jackson, Kenneth, 354, 362

Jefferson, Thomas, 217

Jenkinson, Clay S., book co-edited by, 388-89

Johnson, Colin, 273

Johnson, Doyle "Nig," 274-78

Johnson Foundation, 163

Johnson, Lyndon, 359

Johnson, Russell, 327-28 
Johnson, Sarah, 48-49

Jones, Edith Kathleen, 131-32, 146

Journal of Physical Education, 63-64

Jung, Partick J., book by, reviewed

84-85; book review by, 290-92

Kansas Baseball, 1858-1941, reviewed, 95-96

Kansas-Nebraska Act, 10, 12

Karamanski, Theodore J., book review by, 384-86

Kehrberg, Richard F., book review by, 93-94

Kelderman, Frank, book by, reviewed, 190-92

Keller, Ron J., book by, reviewed, 8990

Kennan, George F., 157

Kennon, Donald R., book co-edited by, reviewed, 93-94

Kett, Joseph, 313-15, 330

Khrushchev, Nikita, 345

Kingsley, Allen, 49-52

Kinne, La Vega, G., 125, 128

Kitchen debate, 345-46

Klumpp, Andrew, article by, 1-34

Know Nothing Party, 9, 11, 13

Koerselman, Rebecca A., book review by, $400-01$

Konrád, György, 180

Korean War, 74

Kramer, Paul, 159

Kretschmer, Herbert, 326

Ku Klux Klan, 258-68, 274, 277, 27981

Kutzler, Evan, book by, reviewed, 298-300

Lahlum, Lori Ann, book co-edited by, reviewed, 201-03

Lake Minnetonka, Minnesota, 223

Lakota America: A New History of Indigenous Power, reviewed, 286-88

László, Szabolcs, article by, 153-85

Lauck, Jon K., book edited by, reviewed, 285-86

Le Cocq, Francis, Sr., 29-30

Leaving home (adolescents), 328-30

Lee, R. Alton, book co-authored by, reviewed, 393-95

Leggett, James, 237-38
Lehman, Christopher P., book by, reviewed, 378-80

Lenderink, Henry J., 30-33

Letsos, Niko, book review by, 398400

Levitt, William, 366

Levittown, New York, 358-59

Lewis, Mary, 313-14

Librarianship, professionalization of, 122-51

Library Bill of Rights, 148

Library Services and Reconstruction Act (Title IV-A), 150

A Life on the Middle West's Never-Ending Frontier, reviewed, 203-05

Lincoln, Abraham, 12

Lincoln in the Illinois Legislature, reviewed, 89-90

Lincoln's Confidant: The Life of Noah Brooks, reviewed, 91-92

Lindell, Lisa, article by, 121-51

Little, Jim, 255-58

Living by Inches: The Smells, Sounds Tastes, and Feeling of Captivity in Civil War Prisons, reviewed, 298300

Lofaro, Michael A., book edited by, reviewed, 290-92

Lomax, Michael E., book review by, 95-96

Lost Cause, 220, 230

Lotte, A. Morris, 150

Lyftogt, Kenneth L., book review by, 92-93

Mack, Jennifer E., article co-authored by, 311-41

Madison, James, 217

Mallea, Amahia, book review by, 369-71

Manhattan Project, 349

Manifest Destiny, 352

Marathon, Iowa, 263

Marietta Academy, 59-60

Marietta, Ohio, 58-60

Marion County, Iowa, 11-12

Markley, James E. E., 239

Marriage, age at, 317-20; graph showing marriage age, 319

Marshall Plan, 156

Martin, Archie, 360-61 
Martin, Nancy, 360-61

Masculinity, see rural masculinity

Mason City Globe-Gazette, 217, 234

Mason City, Iowa, 211-46

Mason, Kevin, book review by, $198-$ 99

Massachusetts compulsory school law, 313

Massacre in Minnesota: The Dakota War of 1862, the Most Violent Ethnic Conflict in American History, reviewed, 380-82

Maulden, Kristopher, book by, reviewed, 288-90

McCloy, Charles Harold, 56-83; image of, 61, 77

McConnell, Stuart, 215

Memoirs as primary source material, 249-50

Memorial College (Oberlin, KS), 220-21

Memorial University, 211-46; alternate locations for, 222-24; enrollment, 235-38, 241, 243; image of envisioned plan, 216; image of first academic building, 225; normal department, 240-41

Meningitis, 331

Men's Reformatory at Anamosa, 129, 139-40, 144

The Merchant Prince of Black Chicago: Anthony Overton and the Building of a Financial Empire, reviewed, 39193

Mergenthal, Rebekah M.K., book review by, 367-69

Mexican migrant workers, 255-58

Mexico Border Traveling Library Service, 146

Middle-class ideals, 312-13

Military drill, 232-33, 236

Military instruction, 233-34

Miller, Benjamin L., book by, reviewed, 194-95

Miller, Char, book co-edited by, reviewed, 388-89

Mills County, Iowa, 35

Ministry of Culture (Hungary), 163

Ministry of Interior, 164-65
The Misunderstood Mission of Jean Nicolet: Uncovering the Story of the 1634 Journey, reviewed, 84-85

Mississippi River, 176, 184, 333

Mitchell, Robert B., book review by, 91-92

Mitton, Frank, 327

Moline, Illinois, 162, 176

Moore, Jane Ann, book co-authored by, reviewed, 375-76

Moore, William F., book co-authored by, reviewed, 375-76

The Mormon Handcart Migration: "Tounge nor pen can never tell the sorrow," reviewed, 294-96

Morrill Act, 232

Morris, Betty Lee, 349

Morris, Edward, 346, 348-49, 352-57; image of, 348

Morris, Gertrude (Rutheford), 34647

Morris, Leslee, 349, 357-58, 363

Morris, Mary Jane, 349

Morris, Neva, 343, 346-49, 357-58, 364-66; image of, 348; image of home, 350, 365

Morris, Walter, 346-47

Morris, Walter L., 348-49

Moscow, USSR, 159, 171,

Moulton, Candy, book by, reviewed, 294-96

The Mound Builder Myth: Fake History and the Hunt for a "Lost White Race," reviewed, 371-73

Mount Pleasant Mental Health Hospital, 129, 140

Muscle-boundedness, 64-67, 76-77, 80

Museum of Modern Art, 157

Musical diplomacy, 156-58, 178

Murphy, J.T., book review by, 95-96

Naegele, Daniel, book review by, 390-91

National Animal Disease Laboratory, 351,360

National Collegiate Athletic Association, 73, 82

National Football League, 74

National Tribune, 218, 221 
National university, 217-22, 227, 235, 240-41

Native Americans, 7-8, 18

Nelson, Lawrence J., book co-authored by, reviewed, 109-11

Nemes Nagy, Ágnes, 171-72, 177, 179

The Netherlandish Association for Emigration to the United States of North America, 4-5

The Netherlands, 3-7, 15, 17

Neu, Jonathan, article by, 211-46

New Deal legislation, 365

New England, 313-14, 320

New Jersey, 322

New York City, 248

New York State Library Association, 131

Nicholson, Ben, book co-edited by, reviewed, 308-10

Nicols, Roger L., book review by, 84 85

Nieh, Hualing, 160, 178, 181; image of, 163

Nikita Khrushchev's Journey into America, reviewed, 109-11

Nixon, Richard, 345

North Star Rural Club, 347

North Star School, 362

Northwood, 359

Oberlin, Kansas, 221-22

O'Connor, Frank "Bucky," 69-70

O'Connor, Henry, 43-44

Offenburger, Andrew, book review by, $185-87$

The Old Curiosity Shop, 141

Old-Fashioned Modernism: Rural Masculinity and Midwestern Literature, reviewed, 396-98

Oldt, Franklin T., 323; biographies by, 323-25, 328-29

Oler, Andy, book by, reviewed, 39698

Olympic games, 75

Olympic weightlifting, 66

Ontario, Iowa, 351

Onymousness, 248-50, 254-55, 250, $257-58,267-68,273-81$

Orange City, Iowa, 19-34

Orbán, Ottó, 172, 174, 179
Orton, Chad M., book review by, 294-96

Ouellette, Richard D., book review by, 292-94

Outsiders in rural Iowa, 254, 255-59, 278,280

Owen Lovejoy and the Coalition for Equality: Clergy, African Americans, and Women United for Abolition, reviewed, 375-76

Pacific Railway Act, 15

Park View Heights, 343-66

Parker, Francis W., 228

Parrillo, Nicholas, 267

Patrick, Jeffrey L., book by, reviewed, 92-93

Patriot's Hill, 212

Patton, Walter J., 239-44

Paul, R. Eli, book edited by, reviewed, 303-05

Payne, Harvey, book co-authored by, reviewed, 113-14

Peck, Graham A., book review by, 375-76

Pegram, Thomas R., book by, reviewed, 102-04

Pella, Iowa, 3-4, 10-22

Pelmulder, Jelle, 28-30

Pendleton, Charles, 247-83

People's Convention, 27-29

People's Daily, 182

Peoples' Friendship University, 159

Peppermint Kings: A Rural American History, 386-88

Philadelphia, 334

Physical Culture, 59, 62, 65

Physical education, 56-83

Physical Educator, 71, 81

Physical training, 62-64

Pierce, James O., 228-29

Plagued by Fire: The Dreams and Furies of Frank Lloyd Wright, reviewed, 390-91

Plainfield, New Jersey, 223

Plummer, Mary Wright, 131

Poland, 162, 164, 181

Poles in Illinois, reviewed, 306-08

Poll tax, 314

Popescu, Petru, 180

Pragatz, B., 332 
The Preacher's Wife: The Precarious Power of Evangelical Women Celebrities, reviewed, 400-01

President's Council on Youth Fitness, 75

Prifogle, Emily, article by, 247-83

"'Product Patriots As Well As Scholars': GAR Educational Reform and the Establishment of Mason City's Memorial University," 21146

Private land ownership, 344, 354, 366

Progress, postwar definitions of, 343-47, 349-50, 352-53, 366

Prohibition, 9, 13, 259, 262-68, 279

Prohibition, the Constitution, and States' Rights, reviewed, 100-02

Prosecutorial discretion, 247-83; distinction from discretion, 251

Queerness in rural Iowa, 273-80

Qian, Xiao, 182

Racial discrimination, 157, 345, 36062

Radzilowski, John, book co-authored by, reviewed, 306-08

Railroads, 15, 29, 58

Rape, laws related to victims, 49-52

Raymond, C. Elizabeth, book review by, 396-98

Raymond, Levi B., 228-29

Reconciliationism (Civil War), 216, 246

Rees, Amanda, book reviewed by, 285

The Reformatory Press, 139

Remembrance of Things Present: The Invention of the Time Capsule, reviewed, 104-06

The Republic Magazine, 243

Republican Party, 10-17, 25-34, 4142

Republican political theory, 39-40, 53-54

Research Quarterly, 67-68, 79

Return to the City of Joseph: Modern Mormonism's Contest for the Soul of Nauvoo, reviewed, 87-89

Richards, Benjamin, 54

Richardson, John, 167-68, 178
Richmond, Heather, book co-authored by, reviewed, 98-100

Riley, Glenda, 318-19

Rittenhous, Rufus, 322-23, 326, 330$32,334-35,340$

Roberts, Tim, book review by, 301-03

Robinson, Gifford S., 125, 132-33

Robinson, Julia, 133-35, 143-46

Rockefeller Foundation, 161, 169

Romania, 164, 180-81

Romeo, Sharon, article by, 35-55

Ronda, James P., book co-authored by, reviewed, 113-14

Roosevelt neighborhood (Ames), image of, 359

Roosevelt, Theodore, 227, 235

Rothert, Henry W., 128

Rowe, S. Frances, 144

Rozum, Molly P., book co-edited by, reviewed, 201-03

Rudolph, Frederick, 214

Rural masculinity, 253, 259-60, 265, 268-73, 278-80, 283

The Rural Schools of Madison County: A Vanishing Heritage, reviewed, 395-96

Rural social networks, characteristics of, 249, 255; examples of, 255-81

Sabatino, Michelangelo, book co-edited by, reviewed, 308-10

Salafia, Matthew, book review by, 196-97

Sargent, Dudley, 60

Savery, Annie, 47, 54

Scholastic Coach, 78

Schaupp, John M., 356

Scholte, Henry, 3-5, 8, 11-14

School attendance rates, 314, 317, 320-24, 336-37, 341; graph of, 337

School for the Blind, 129

School of the Deaf, 129-29

Schwieder, Dorothy, 259-60, 364-65

Second Great Awakening, 316

Seducible women, 35-55

Seduction Laws, 35-55

Seevers, William, 47-48

Servicemen's Readjustment Act, 71$72,350,366$

Settler colonialism, 8

Shean, Andrew, 43-47 
Shellenberger, Grace, 133, 140-41, 146,148

" "The Shock of Seeing the Freedom of American Life,': The Iowa International Writing Program as Cultural Diplomacy during the Cold War," 153-85

Shoenbachler, Matthew G., book cowritten by, reviewed, 109-11

"The Short American Century," 346

Shorthorn cattle, 347

Shurley, Jason, article by, 56-83

Silver, Timothy, book co-authored by, reviewed, 384-86

Sioux City Journal, 22

Sioux County, Iowa, 1-34

Sioux County Board of Supervisors, 21-22, 26-27

Sioux County Herald, 24, 26-28, 31-33

Sioux County Independent, 30

Sioux Rapids, Iowa, 253, 263

Sipma, Sjoerd, 15

Skunk River, 352

Slavery, 11, 13, 220, 230

Slavery's Reach: Southern Slaveholders in the North Star State, reviewed, 378-80

Smith, Zachary, book by, reviewed, 100-02

Smithsonian Institute, 223

"'So Well Begun and So Much

Needed': Building up Libraries for Residents of Iowa's State Institutions," 121-51

Social worker in Buena Vista County, 268-70

Society of Christians for the Holland Emigration to the United States of North America, 4-5

Soldiers' Home, 128-29

Sons of Veterans, 216, 219, 222, 224$25,232,239,243-45$

Sortor, Alexander Louis, Jr., 222, 225$28,234,236,240$

Soviet Bloc, 163-65, 183

Soviet propaganda, 155-56

Soviet Union, 74-75, 155, 159, 164, 171

Spalding, A. G., 58

Spanish-American War, 214, 222; veterans of, 211
Spaulding, Forrest, 148

Spirit Lake Massacre, 8

Spotted Tail: Warrior and Statesman, 198-99

Spragg, Dennis M., book by, reviewed, 398-400

Squaw Creek, 352

St. Louis, 329

Stanton, Elizabeth Cady, 43

State Hospital for Inebriates, 130, 138,144

State Library Board, 125

State Normal and Industrial College for Colored Students (Tallahassee, Florida), 237

State v. Bell, 35-42, 53

State v. Carron, 36, 44-46, 53

State v. Gardner, 38-39

State v. Haven, 36, 47-49, 53

State v. Kinglsey, 36, 49-53

State v. Tarr, 52

Stek, Pam, book review by, 201-03

Stinson, Jennifer K., book review by, 378-80

Stockett, Julia Carson, 133, 135, 14042, 146, 149-50; image of, 142

Stone, Fred, 31-33

Stone, Rufus, 24, 31

Storm Lake, Iowa, 247-83

The Storm Lake Register, 264

Story County, Iowa, 343, 346-48, 351, 356,363

Story County Fair Association, 347

Story County Land Trust, 362

Strength training, 64-83; see also weight training.

Strength $\mathcal{E}$ Health, 65, 76, 81

Suburbanization, 343-66

Such Anxious Hours: Wisconsin

Women's Voices from the Civil War, reviewed, 300-01

Suffragists, $41-43$

Svaty, Joshua L., book co-authored by, reviewed, 189-90

Szász, Imre, 165, 172, 174, 178, 181

Taxpayer's Committee, 353-55

Taylor, Amy Murrell, book by, reviewed, 196-97

Taylor, Mildred Dodd, 366

Temperance, 253, 255, 258-59, 262-68 
Temple, Wayne C., book by, reviewed, 91-92

Tennessee, 332, 334

Torrance, Eliakim "Ell," 212, 228-29

Townsend, Eliza E., 133-34, 138-39

Tractor and Gas Engine Review, 347

Transplanters on the Grasslands and the Fruits of Chain Migration, reviewed, 199-201

Tuberculosis, 331-32

Tucker, Frederick D., 237-39

Twain, Mark, 137, 141

Typhoid fever, 331

Tyler, Alice S., 124-28, 134; image of, 124

Undeveloped land, definitions of, 344, 352-53, 358

Union Army, 213, 311, 327

Union Cause, 220, 244-45

Unkultur, 156

U.S. Bureau of Educational and Cultural Affairs, 161, 167

U.S. Department of the Interior, 242

U.S. Information Agency, 158, 161, 177,182

U.S. State Department, 157, 161-62, $165,167,176-78,182$

U.S. Supreme Court, 356

University of Iowa, 56, 58, 61, 64-83

Up from Slavery, 141

Urban imperialism, 354

Utica, New York, 222-23

Vail, David D., book by, reviewed, 108-09

Van Raalte, Albertus, 4-5, 8-12

Veterans of Foreign Wars, 245

Vigilantism, 260, 262-68

Visions of the Tallorass: Prairie Photographs by Harvey Payne, reviewed, 113-14

Voelker, Christian, 325

Vogel, Otto, 70-71

Voice of America, 182

Voldeng, Mathew Nelson, 137

De Volksvriend, 19, 28-30, 33

Volunteers in libraries, 143-45

Wage work, 314, 316, 328
Wagner, R. Richard, book by, reviewed, 96-98

Walczynski, Mark, book by, reviewed, 373-75

Wanko, Andrew, book by, reviewed, 369-71

Warsaw, Poland, 165

Washington, Booker T., 141

Washington, D. C., 157, 160, 165-67, 223, 247-48

Washington, George, 212, 217-18

Water Tower Place, 363

Weems, Robert E., Jr., book by, reviewed 391-93

Weight training, 56-58, 64-83; images of, 72-73; see also strength training

Wendler, Arthur, 65-66, 68-70

Wesley, Timothy, book review by, 194-95

West Coast, 345

West Germany, 156

West Point, 361

Western Reserve Library School, 134

We've Been Here All Along: Wisconsin's Early Gay History, reviewed, 96-98

When Sunflowers Bloomed Red: Kansas and the Rise of Socialism in America, reviewed, 393-95

Whigs, 9-11

Wiebe, Robert H., 215

Wiggin, Kate Douglas, 141

Wilkerson, Miranda E., book co-authored by, reviewed, 98-100

Winkle, Kenneth J., book review by, 89-90

"Winks, Whispers, and Prosecutorial Discretion in Rural Iowa, 192528," 247-83

Wise, Mary, book review by, 371-73

Woman suffrage, 41-44, 47, 53-54, 245

Woman's Christian Temperance Union, 54

Woman's Relief Corps, 216, 219, 224, 227, 235, 239-42, 245-46

The Women's Fight: The Civil War's Battles for Home, Freedom, and $\mathrm{Na}$ tion, reviewed, 382-84

Women's Reformatory, 143 
Wood, Sharon, book review by, 8587

Work related accidents, 332

Worker, Georgia B., 224

"Working Hard and Living Out: Adolescence in Nineteenth-Century Dubuque," 311-41

The World Comes to Iowa, 181-82

World War I, 232, 247

World War II, 249, 349, 365

Writers' Union (Hungary), 163

Wuthnow, Robert, 6

Wynn, Gregory A., book review by, 388-89

Yablon, Nick, book by, reviewed, 104-06

Yankee Bourgeoisie, 312

Yankton College, 60

YMCA, 60-61

Yoder, Franklin, book review by, 395-96

Young brides, 318-19, 339

Yugoslavia, 164

Zieke, Paul, 276 
EACH ISSUE of The Annals of Iowa brings to light the deeds, misdeeds, and accomplishments of our predecessors and shows how they fit into the intricate mosaic of Iowa's past. Its in-depth articles will satisfy even the most serious explorer of Iowa's past.

Anyone with a serious interest in Iowa history will gain valuable perspective from the pages of the Annals. Give it as a gift to a friend or relative. Check to see if your public, school, or academic library subscribes; if they don't, encourage them to do so or, better yet, donate a subscription.

8
Annals of Iowa Subscription
$\square$ New $\square$ Renewal $\square$ Gift* $^{*}$
$\square$ One year, $\$ 24.95$
$\square$ Two years, $\$ 44.95$
$\square$ Three years, $\$ 64.95$

Name

Address

City State Zip

Make check payable to the State Historical Society of Iowa and return with this coupon (or a photocopy of it) to:

Subscriptions

State Historical Society of Iowa

600 E. Locust Street

Des Moines, Iowa 50319

$\square$ Please send information on membership in the State Historical Society of Iowa.

*For gift subscriptions, write the recipient's name and address on this form, and include your name and address on the back or on a separate sheet of paper. Also indicate how you would like your gift card signed. 


\section{Contributors}

JENNIFER E. MACK is a bioarchaeologist with the Office of the State Archaeologist at the University of Iowa. A specialist in historical cemetery research, Jennifer coauthored Dubuque's Forgotten Cemetery: Excavating a Nineteenth-century Burial Ground in a Twenty-firstcentury City (University of Iowa Press, 2015). She is currently completing a PhD at the University of Exeter.

DUSTIN S. CLARK is a field archaeologist with the Office of the State Archaeologist at the University of Iowa. He has seventeen years of experience excavating both prehistoric and historic period sites across the Midwest and the southeastern United States.

EDITH M. HUNTER is a graduate student in the Department of History at Iowa State University. Her research emphasis is $20^{\text {th }}$ Century American social and cultural history with a proposed thesis topic focusing on the loss of war memorials during the scrap metal drives of World War II. In addition to working as an accountant at Iowa State, she also serves as a member of the Ames Historic Preservation Commission. 


\section{The State Historical Society of lowa}

The Annals of Iowa is published quarterly by the State Historical Society of Iowa, the Historical Division of the Department of Cultural Affairs of the State of Iowa. The society operates from two centers, Des Moines and Iowa City. A museum, research library, state archives, special collections, community programming, publications, historic preservation, and membership programs are located at 600 East Locust Street, Des Moines, IA 50319, phone 515-2815111. A research library, and special collections are located at 402 Iowa Avenue, Iowa City, IA 52240, phone 319-335-3916. The society also operates several historic sites across the state.

\section{Subscriptions}

Subscriptions to The Annals of Iowa are \$24.95 per year; single copies are \$7. Contact Publications, State Historical Society of Iowa, 600 East Locust Street, Des Moines, Iowa 50319.

The Annals is available on microfilm from Xerox University Microfilms, 300 N. Zeeb Road, Ann Arbor, Michigan 48106.

\section{Submissions}

The Annals of Iowa invites the submission of articles on Iowa history and on subjects concerning the nation and the Midwest with an Iowa focus. State, local, and regional studies of political, economic, social, cultural, intellectual, institutional, ethnic, religious, material culture, archeological, and architectural history are welcome. The Annals also reviews significant books on related topics. A detailed set of editorial guidelines is available on request. All correspondence concerning editorial matters should be addressed to:

Andrew Klumpp, editor

The Annals of Iowa

State Historical Society of Iowa

600 East Locust Street

Des Moines, Iowa 50319

The Annals of Iowa is a participating member of the Conference of Historical Journals.

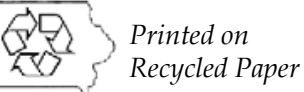

The acid-free paper used in this publication meets the minimum requirements of (O) the American National Standard for Information Services - Permanence of Paper for Printed Library Materials, ANSIZ739.48B1984. 


\section{THE ANNALS OF IOWA}

State Historical Society of lowa

600 E Locust Street

Des Moines IA 50319

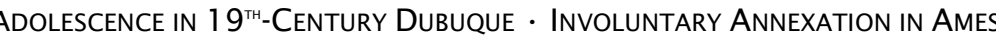

Non-profit Org. .S. Postage

Des Moines, IA 